Article

\title{
Application of the Terrestrial Laser Scanning in Slope Deformation Monitoring: Taking a Highway Slope as an Example
}

\author{
Chao Yin ${ }^{1,2}$, Haoran $\mathrm{Li}^{3}$, Zhinan $\mathrm{Hu}^{2}$ and Ying $\mathrm{Li}^{1, *}$ \\ 1 School of Civil and Architecture Engineering, Shandong University of Technology, Zibo 255000, China; \\ yinchao@sdut.edu.cn \\ 2 Key Laboratory of Roads and Railway Engineering Safety Control of Ministry of Education, \\ Shijiazhuang Tiedao University, Shijiazhuang 050043, China; huzhinan@stdu.edu.cn \\ 3 Urban Rail Construction Corporation, Zhongtian Construction Group Co., LTD, Hangzhou 310000, China; \\ lihaoran@sdut.edu.cn \\ * Correspondence: liying@sdut.edu.cn; Tel.: +86-135-1864-7815
}

Received: 22 February 2020; Accepted: 10 April 2020; Published: 18 April 2020

\begin{abstract}
Slope deformation monitoring is the prerequisite for disaster risk assessment and engineering control. Terrestrial laser scanning (TLS) is highly applicable to this field. Coarse registration method of point cloud based on scale-invariant feature transform (SIFT) feature points and fine registration method based on the k-dimensional tree (K-D tree) improved iterative closest point (ICP) algorithm were proposed. The results show that they were superior to other algorithms (such as speeded-up robust features (SURF) feature points, Harris feature points, and Levenberg-Marquardt (LM) improved ICP algorithm) when taking the Stanford Bunny as an example, and had high applicability in coarse and fine registration. In order to integrate the advantages of point measurement and surface measurement, an improved point cloud comparison method was proposed and the optimal model parameters were determined through model tests. A case study was conducted on the left side of the K146 + 150 point at S236 Boshan section, Shandong Province, and research results show that from 14 August 2018 and 9 November 2019, the overall deformation of the slope was small with a maximum value of $0.183 \mathrm{~m}$, and the slope will continue to maintain a stable state without special inducing factors such as earthquake, heavy rainfall and artificial excavation.
\end{abstract}

Keywords: terrestrial laser scanning (TLS); slope deformation monitoring; point cloud registration; improved point cloud comparison method

\section{Introduction}

The original stress state of natural or artificial slopes will change in the process of geological evolution and engineering construction, resulting in stress redistribution and stress concentration [1]. In order to adapt to the new stress state, the rock and soil of slope will undergo different forms and scales of deformation (such as unloading rebound and creep) [2-4]. Revealing the probability and extent of disastrous deformation are the prerequisites for slope stability analysis [5]. Traditional slope deformation monitoring methods include manual survey, global navigation satellite system (GNSS) measurement, total station measurement and close-range photogrammetry [6]. Among them, manual survey is unsuitable for high and steep slopes, and the results depend on human interpretation of the terrain, which is highly subjective [7]; GNSS measurements and total station measurements are single-point measurement methods, which have difficulties in determining the deformation of the areas without monitoring points, moreover, once the monitoring points are destroyed, the continuity of the data will be affected $[8,9]$. In close-range photogrammetry, each image covers only a small part of the 
scene and the data processing involves cumbersome processes such as orienteering and inlay, resulting in low work efficiency $[10,11]$. Slope deformation monitoring based on terrestrial laser scanning (TLS) can provide discrete three-dimensional data of the slope surface, thereby avoiding the locality and one-sidedness of stress-strain analysis based on data from single monitoring point [12]. These advantages ensure that TLS-based slope deformation monitoring has broad application prospects $[13,14]$.

Affected by factors such as the performance of TLS facility and range of slope, the whole information of some slopes needs to be acquired through multi-station scanning, and the point cloud data of each station need to be registered to the same coordinate system [15,16]. Point cloud registration includes feature-based registration and featureless registration [17], of which feature extraction is the core of the feature-based registration algorithm, and representative studies include: Zhu et al. [18] proposed an image registration algorithm named belief propagation (BP)-scale-invariant feature transform (SIFT), where the keypoint matching of SIFT descriptors was formulated as a global optimization problem and provided a suboptimum solution using BP; Chen and Shang [19] presented an improved SIFT algorithm on characteristic statistical distributions and consistency constraints since the feature descriptor section of conventional SIFT does not make full use of the local feature information; Wang et al. [20] proposed an algorithm for automatic extraction of diagonal marks by combining template matching and gradient peaks, which improved the robustness of corner point detection; Lu et al. [21] proposed a super 4-points congruent sets (4PCS) algorithm combining the scale invariant features in light of the low efficiency of the traditional initial registration method; and Ran and $\mathrm{Xu}$ [22] proposed a point cloud registration method based on SIFT and geometry feature in order to improve the registration accuracy. The object was extracted based on threshold segmentation, the initial corresponding points were acquired based on the SIFT operator, and furthermore, the curvature was performed to remove the wrong points.

Besl and McKay [23] proposed the iterative closest point (ICP) algorithm, which by far is the most widely used featureless point cloud registration algorithm. It calculates the rotation and translation transformation matrix of the point cloud to be registered by creating a corresponding point pair with the smallest Euclidean distance between the reference point cloud and point cloud to be registered [24]. The ICP algorithm needs to fulfill two basic conditions: (1) There is a common area between the reference point cloud and point cloud to be registered; and (2) The initial relative deviation between the reference point cloud and point cloud to be registered is not large [25,26]. Some scholars have improved the ICP algorithm and representative studies include Wei et al. [27], who proposed an ICP algorithm based on point cloud homography and explained the method of establishing homography point pairs; $\mathrm{Li}$ and Song [28], who proposed a vector information registration method based on facet triangles in the stereolithography (STL) format file and introduced a dynamic adjustment factor to speed up the iterative convergence; Cheng et al. [29] proposed an improved ICP algorithm, which used a point-to-point correspondence instead of point-to-surface correspondence to reduce the calculation cost; Wu et al. [30] proposed a novel robust scale ICP algorithm by introducing the maximum correntropy criterion (MCC) as the similarity measure when the point sets had a large number of outliers and noises; Combès and Prima [31] presented an efficient expectation-maximisation (EM)-ICP algorithm for non-linear pairwise registration of large 3D point sets, compared to other methods using the same "EM-ICP" framework, four key modifications leading to an efficient algorithm: (1) truncation of the cost function; (2) symmetrization of the point-to-point correspondences; (3) specification of priors on these correspondences using differential geometry; and (4) efficient encoding of deformations using the reproducing kernel Hilbert space (RKHS) theory and the Fourier analysis.

Slope deformation measurement based on TLS determines the spatial position of the comparison point relative to the reference point by processing the reference point cloud (point cloud of the first phase) and the comparison point cloud (point cloud of the second phase) [32]. It includes four types of methods: point cloud superposition method, point cloud comparison method, center of gravity method, and fitting method [33]. Among them, the point cloud superposition method and point cloud comparison method belong to surface measurement as their monitoring points do not necessarily 
correspond to one-to-one, and the center of gravity method and fitting method can achieve one-to-one correspondence of monitoring points, equivalent to conventional feature point measurement [34]. Representative studies include Schürch et al. [35], who realized landslide stability monitoring by calculating the landslide volume in different periods based on the point cloud and digital elevation model (DEM); Zhu et al. [36] proposed a method for surface deformation monitoring of high-risk slopes based on TLS that extracted the normal vector of the reference points and calculated the center of gravity of the comparison point cloud, which were used as the basis for comparison of surface deformation; and Hu et al. [37] took the front edge of Jinpingzi landslide II region as the research object, arranged target bodies in "surface monitoring areas" and performed deformation analysis on the target bodies.

Slope deformation monitoring based on TLS can still be improved in the following aspects:

(1) Regarding point cloud registration, existing feature extraction algorithms have disadvantages such as low extraction efficiency, require multiple parameters, and have poor real-time performance for large scenes; existing improved ICP algorithms have disadvantages such as high time and space complexity and are prone to matching errors and slow iterative convergence;

(2) Regarding slope deformation measurement, point measurement methods have high accuracies, but only the key parts can be monitored and the deformations in areas without monitoring points cannot be revealed, whereas surface measurement methods can describe the deformation of each part of the slope surface, but the accuracies are low. Thus, a slope deformation measurement method integrating the advantages of point measurement and surface measurement does not yet exist.

In light of this, the slope on the left side of the K146 + 150 point at S236 Boshan section, Shandong Province was taken as the research object and the point clouds were collected by TLS, coarse registration of point cloud based on SIFT features and fine registration based on k-dimensional tree (K-D tree) improved ICP algorithm were conducted respectively, and the slope deformations were calculated by the improved point cloud comparison method with the aim to provide a direct basis for disaster risk assessment and engineering control.

\section{Point Cloud Data Registration Method}

\subsection{Methodology}

The goal of point cloud coarse registration is to reduce the rotation and translation dislocation between the reference point cloud and point cloud to be registered, and provide a good initial value for fine registration $[17,31]$. The goal of fine registration is to obtain the optimal registration parameters and minimize the errors [38]. In this paper, the corresponding point pairs were obtained by extracting the SIFT features, the error point pairs were eliminated based on the random sample consensus (RANSAC) algorithm, the initial matrix was determined by utilizing the singular value decomposition (SVD) algorithm to complete the coarse registration. In order to improve the overall point cloud data registration speed, K-D tree was used to search for the nearest point in the ICP algorithm to complete the fine registration, as shown in Figure 1.

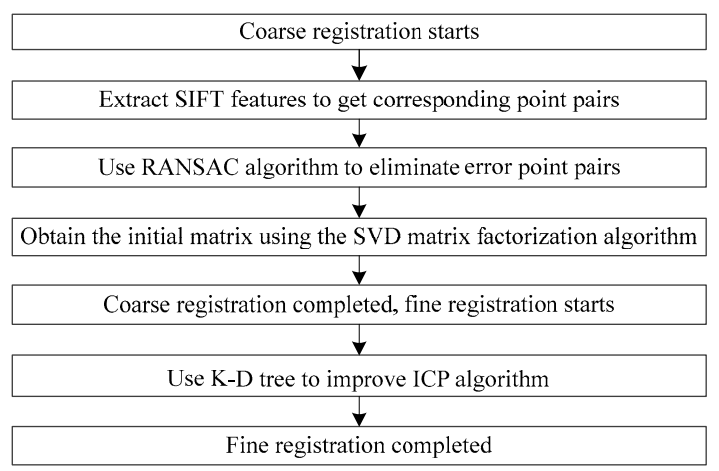

Figure 1. Point cloud data registration method. 


\subsubsection{SIFT Feature Extraction}

SIFT feature extraction includes spatial feature point extraction, feature point detection, determination of the feature point direction, and determination of the feature point descriptor [39]. The main processes include:

Assume that the coordinate of a point in the point cloud is $P(x, y, z)$ and the spatial scale of the point cloud is $L(x, y, z, \sigma)$. The relationship between the point coordinate and the spatial scale is as shown in Equation (1).

$$
L(x, y, z, \sigma)=G(x, y, z, \sigma) * P(x, y, z)
$$

where $\sigma$ is the spatial scale factor of the point cloud; $G(x, y, z, \sigma)$ is the three-dimensional Gaussian kernel function of the point cloud; and the calculation method is as shown in Equation (2).

$$
G(x, y, z, \sigma)=\frac{1}{(\sqrt{2 \pi} \sigma)^{3}} e^{\frac{-\left(x^{2}+y^{2}+z^{2}\right)}{2 \sigma^{2}}}
$$

When constructing a point cloud Gaussian pyramid, the Different-of-Gaussian (DoG) operators at the $k$ scale are defined as shown in Equation (3).

$$
D\left(x, y, z, k^{s} \sigma\right)=L\left(x, y, z, k^{s+1} \sigma\right)-L\left(x, y, z, k^{s} \sigma\right)
$$

where $s \in[0,1]$. Using the Lowe operator to linearly fit the Gaussian difference-scale space function and eliminate extreme points with strong edge effects, the remaining extreme points are the feature points. Equation (4) is used to calculate the amplitude $m(x, y, z)$, azimuth $\theta(x, y, z)$, and angle $\varphi(x, y, z)$ from the feature point $\left(x_{i}, y_{i}, z_{i}\right)$ to its neighborhood center point $\left(x_{c}, y_{c}, z_{c}\right)$.

$$
\left\{\begin{array}{l}
m(x, y, z)=\sqrt{\left(x_{i}-x_{c}\right)^{2}+\left(y_{i}-y_{c}\right)^{2}+\left(z_{i}-z_{c}\right)^{2}} \\
\theta(x, y, z)=\tan ^{-1}\left(\left(y_{i}-y_{c}\right) /\left(x_{i}-x_{c}\right)\right) \\
\varphi(x, y, z)=\sin ^{-1}\left(\left(z_{i}-z_{c}\right) / m(x, y, z)\right)
\end{array}\right.
$$

Based on the azimuth and angle of the neighborhood of each feature point, define the amplitude as the weight and smooth the histogram, and define the peak direction of the histogram as the feature point direction. Define a four-dimensional vector $(\sigma, m, \theta, \varphi)$ for any feature point, divide its neighborhood into sub-domains of $n^{*} n^{*} n$, and count the frequency of occurrence of all neighborhood points in the sub-domain in each direction. The frequency of occurrence is the feature point descriptor.

\subsubsection{K-D Tree Improved ICP Algorithm}

In the K-D tree improved ICP algorithm, the following methods are used to search the nearest point [40-42]:

(1) Compare the node to be checked with the value of the determined splitting dimension. If the value of the determined splitting dimension is larger, it enters the left sub-tree, and if it is smaller, it enters the right sub-tree. In this manner, loop to the leaf nodes of the binary tree and find the nearest point in the same subspace of the node to be checked along this search path;

(2) Perform a "backtrack" operation for each point. If the other subspaces of the nodes along the search path have closer points, skip to the subspace nodes to search the nearest point; and

(3) Repeat the above two steps until the search path is empty.

\subsection{Verification of the Methodology}

In order to verify the point cloud data registration method, this study took the Stanford Bunny as the research object to perform the coarse registration based on SIFT feature points, SURF feature points, and Harris feature points, and compared the quantity of extracted feature points, accuracy 
rates, and elapsed times [19]. Furthermore, the elapsed times and mean square errors (RMSEs) of the K-D tree improved ICP algorithm and Levenberg-Marquardt (LM) improved ICP algorithm were verified respectively in the fine registration [30].

\subsubsection{Coarse Registration}

Figure 2 shows the feature point extraction effect. Figure 2a shows the initial point clouds at two stations, and each station includes 35,947 sampling points; Figure 2b-d show the extracted SIFT feature points, SURF feature points, and Harris feature points respectively, where the red points are the initial point cloud and the green points are the extracted feature points.

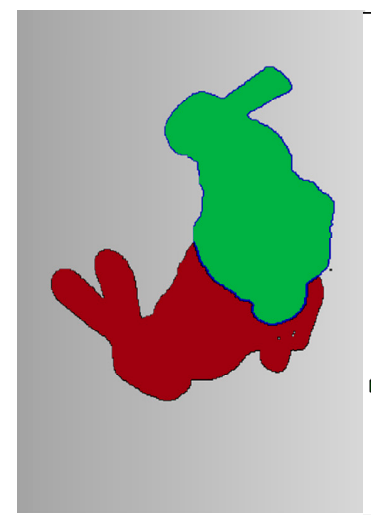

(a)

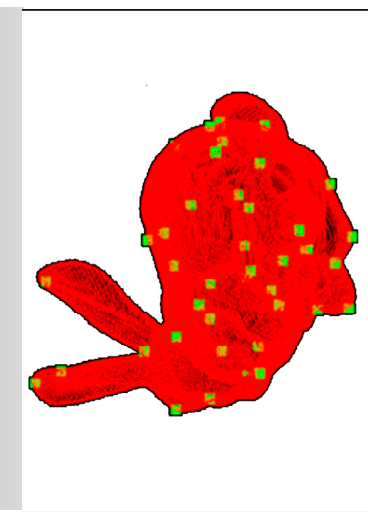

(b)

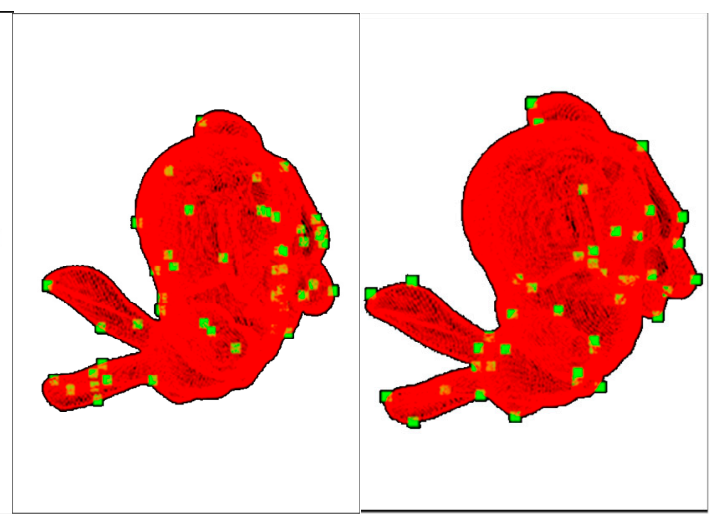

(c)

(d)

Figure 2. Feature point extraction effect: (a) initial point clouds, (b) SIFT feature points, (c) SURF feature points, (d) Harris feature point.

According to Figure 2, the quantity, accuracy rate, and elapsed time of each kind of feature point were calculated respectively, as shown in Table 1.

Table 1. Quantitative indicators for feature point extraction.

\begin{tabular}{cccc}
\hline Feature Point Type & Quantity & Accuracy Rate & Elapsed Time \\
\hline SIFT feature point & 39 & $100 \%$ & $20.37 \mathrm{~s}$ \\
SURF feature point & 41 & $98.6 \%$ & $27.82 \mathrm{~s}$ \\
Harris feature point & 36 & $91.7 \%$ & $25.98 \mathrm{~s}$ \\
\hline
\end{tabular}

As can be seen from Table 1, since the Stanford Bunny is a kind of standard model data and contains no noise [43], both the reference point cloud and point cloud to be registered can find strict points with the same name, therefore, the registration accuracies of the three algorithms were relatively high. Comparably, the extraction accuracy rate of the SIFT feature points reached $100 \%$ and took the least time, and the overall performance was better than the SURF feature points and Harris feature points. Therefore, it is reasonable and feasible to perform point cloud coarse registration based on SIFT feature points.

\subsubsection{Fine Registration}

After applying SIFT feature points to perform coarse registration, fine registration utilizing the $\mathrm{K}-\mathrm{D}$ tree improved ICP algorithm and LM improved ICP algorithm were performed respectively, of which, the LM improved ICP algorithm can solve the problem of slow convergence of the classic ICP algorithm when the Gauss-Newton approximation error is large [44]. The registration results of the two algorithms are shown in Figure 3. 


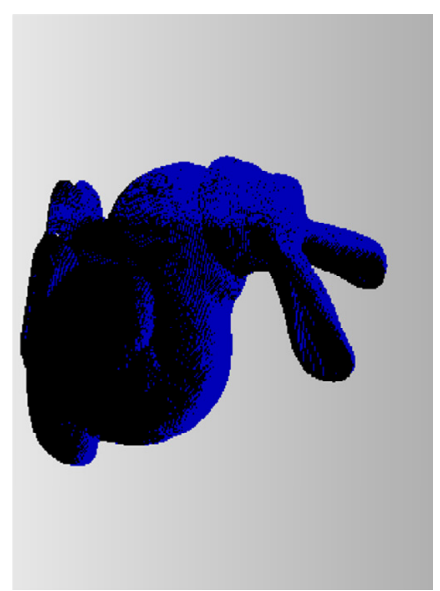

(a)

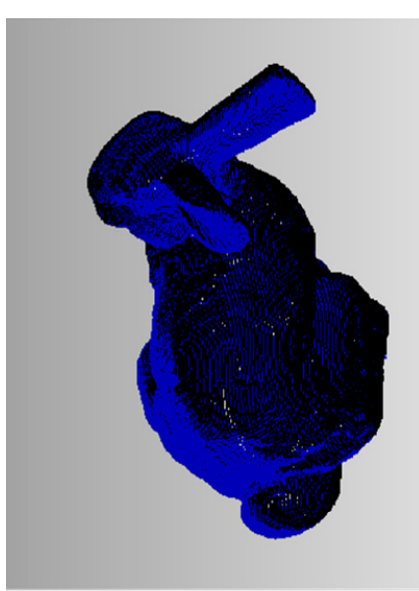

(b)

Figure 3. (a) registration results of K-D tree improved ICP algorithm, (b) registration results of LM improved ICP algorithm.

The advantages and disadvantages of the two algorithms were evaluated by the elapsed time and RMSE; the results are as shown in Table 2. As can be seen from Table 2, the K-D tree improved ICP algorithm was better than the LM improved ICP algorithm. Therefore, it is reasonable and feasible to use the K-D tree improved ICP algorithm to perform fine registration.

Table 2. Quantitative indicators of point cloud data registration.

\begin{tabular}{ccc}
\hline Registration Method & Elapsed Time & RMSE \\
\hline K-D tree improved ICP algorithm & $3.575 \mathrm{~s}$ & $0.445 \mathrm{~mm}$ \\
LM improved ICP algorithm & $5.451 \mathrm{~s}$ & $0.987 \mathrm{~mm}$ \\
\hline
\end{tabular}

\section{Improved Point Cloud Comparison Method}

\subsection{Methodology}

The integration of point measurement and surface measurement can not only accurately measure the deformation of a single monitoring point, but also obtain the overall deformation of the slope surface [32-34,45]. In order to integrate the advantages of point measurement and surface measurement, an improved point cloud comparison method was proposed. This method used the normal vector of the reference point cloud as the axis to construct a prism, and determined the position of the comparison point cloud by calculating the center of gravity in the prism and extracted the three-dimensional deformation information along the normal direction.

The main ideas are as follows [33,46,47]:

(1) For any reference point $i(x, y, z)$, construct a covariance matrix based on the neighborhood point set in the sphere with the reference point as the center and $R$ as the radius, and find the eigenvector corresponding to the smallest eigenvalue, that is, the normal vector $N$ of the reference point;

(2) Construct a prism with the normal vector $N$ as the axis, $d$ as the side length, and $H$ as the height. The prism contains a reference point set and a comparison point set, of which the reference point $i$ is located in the reference point set;

(3) Project the comparison point set onto the normal vector $N$, and calculate the barycentric coordinates of the projected points. The barycentric point is the point of the same name corresponding to the reference point $i$, and the distance of the point pair of the same name is calculated as the deformation amount of the reference point; and

(4) Obtain the model parameters with the highest accuracy of slope deformation measurement through model tests. Conduct study on the research object to obtain the amount of slope deformation 
during the monitoring period based on the optimal parameters. The calculation principle is as shown in Figure 4.

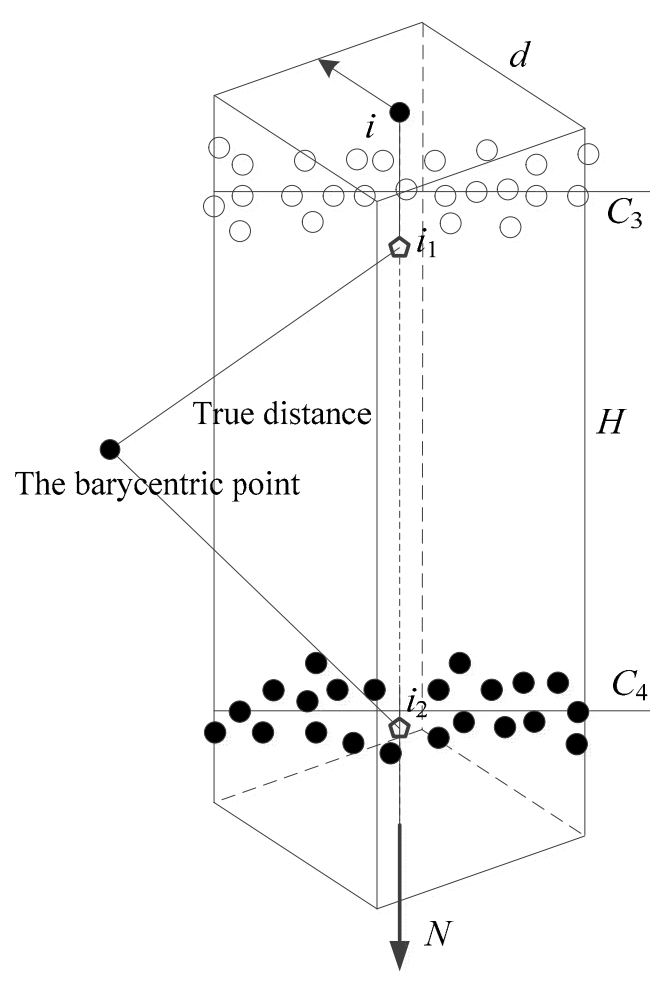

Figure 4. Calculation principle of the improved point cloud comparison method.

It is important to note that differences between the reference point cloud and comparison point cloud may be caused by non-slope deformations such as the growth of vegetation, so it is very important to filter the influence of the vegetation on the deformation measurement. An interesting ground filtering method was developed by Li et al. [48], who proposed a novel filter of LiDAR point clouds based on geodesic transformations of mathematical morphology, where the algorithm made it unnecessary to select different window sizes or determine the maximum window size, which could enhance the robustness and automation for unknown environments. In this paper, this algorithm was adopted in ground filtering before the slope deformation measurement.

\subsection{Model Tests}

In order to assess the applicability of the improved point cloud comparison method and analyze the calculation errors, this study conducted model tests to determine the model parameters with the highest measurement accuracy [49,50].

\section{(1) Test model}

The main body of the test model was a rectangular gray iron plate of $1.2 \mathrm{~m} \times 1.8 \mathrm{~m}$. The inclination of the iron plate was set to $45^{\circ}$ to simulate the slope. A black cone magnet was placed on the iron plate to simulate the slope deformation by moving the position of the magnet. The displacement of the magnet was controlled by a Vernier caliper, as shown in Figure 5 . 


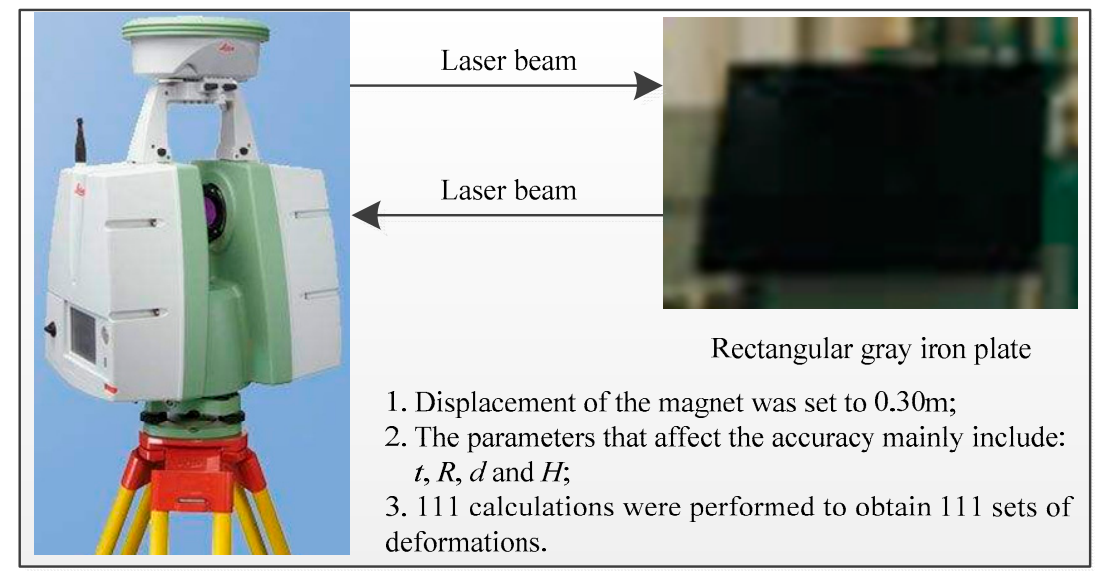

Figure 5. Test model.

\section{(2) Contents of tests}

When conducting the slope deformation measurement, the parameters that affect the accuracy mainly include the interval of scanning points $t$, radius of the sphere used for normal estimation $R$, side length of the prism $d$, and height of the prism $H$ [51,52]. The commonly used values of these parameters are shown in Table 3.

Table 3. Commonly used values of the parameters.

\begin{tabular}{cc}
\hline Model Parameter & Common Values \\
\hline Interval of scanning points $t$ & $0.05 \mathrm{~m}, 0.10 \mathrm{~m}, 0.20 \mathrm{~m}$ \\
Radius of the sphere used for normal estimation $R$ & $0.05 \mathrm{~m}, 0.10 \mathrm{~m}, 0.20 \mathrm{~m}$ \\
Side length of the prism $d$ & $0.10 \mathrm{~m}, 0.20 \mathrm{~m}, 0.30 \mathrm{~m}$ \\
Height of the prism $H$ & $0.15 \mathrm{~m}, 0.30 \mathrm{~m}, 0.60 \mathrm{~m}$ \\
\hline
\end{tabular}

Displacement of the magnet was set to $0.30 \mathrm{~m}$ and two-phase scanning of the test model was conducted when $t=0.05 \mathrm{~m}, t=0.10 \mathrm{~m}$, and $t=0.20 \mathrm{~m}$, respectively. Orthogonal design on $R, d$, and $H$ was performed to obtain 27 combinations and 81 calculations of the deformation for the point cloud were performed, corresponding to each $t$ through the improved point cloud comparison method [33]. Thirty additional calculations were added in the subsequent data analysis stage. A total of 111 calculations were performed to obtain 111 sets of deformations $l$ and errors $n$, where error $n$ was calculated as shown in Equation (5).

$$
n=|l-0.30|
$$

\subsection{Data Analysis}

Taking one parameter as the variable and the other three as fixed parameters to analyze the calculation results [32], there were four cases in total:

(1) Taking the interval of scanning points $t$ as the variable

Taking the average of the 27 sets of calculation results corresponding to a certain $t$ value, and obtaining the relationship between $t$ and $n: t$ and $n$ have a positive correlation, that is, the smaller $t$ is, the smaller $n$ is, but $t<0.05 \mathrm{~m}$ will significantly increase the time consumed for scanning and deformation calculation, reducing the applicability of the model, as shown in Figure 6a. 


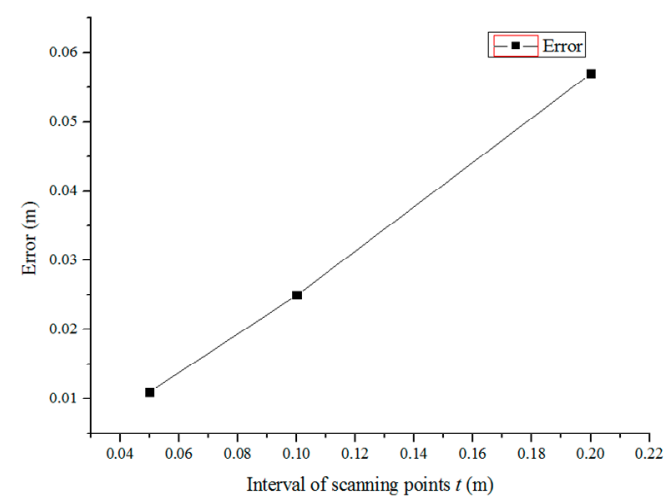

(a)

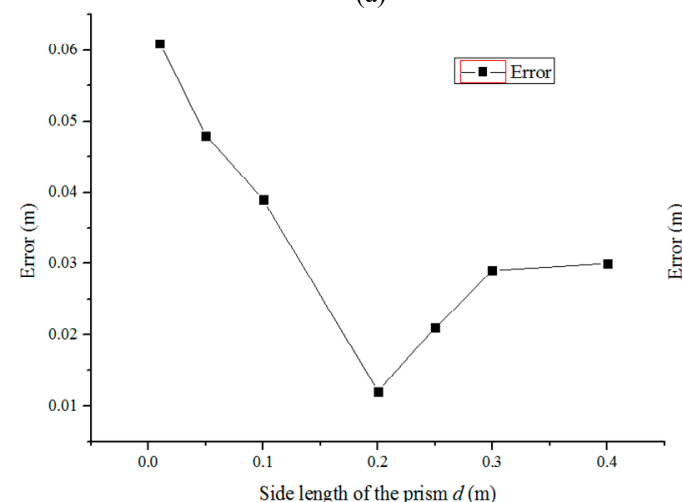

(c)

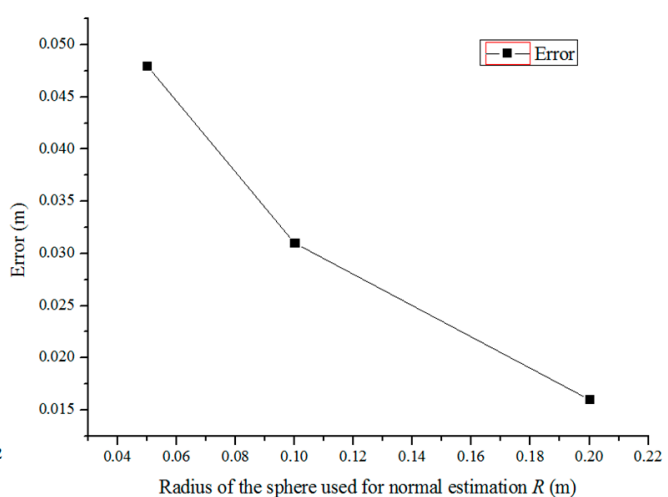

(b)

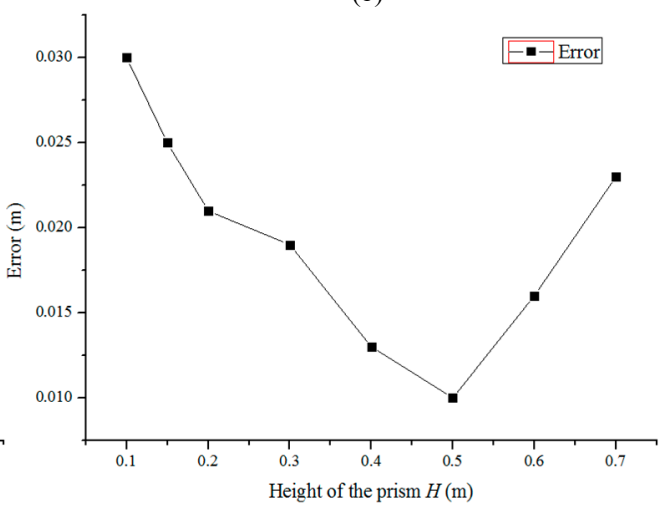

(d)

Figure 6. Data analysis results: (a) relationship between $t$ and $n$, (b) relationship between $R$ and $n$, (c) relationship between $d$ and $n$,(d) relationship between $H$ and $n$.

(2) Taking the radius of the sphere used for normal estimation $R$ as the variable

Similarly, the relationship between $R$ and $n$ is as follows: $R$ and $n$ are inversely correlated, that is, the smaller $R$ is, the larger $n$ is, but $R>0.20 \mathrm{~m}$ will greatly increase the time consumed for deformation calculation, as shown in Figure $6 \mathrm{~b}$.

(3) Taking the side length of the prism $d$ as the variable

Since the relationship between $d$ and $n$ is more complicated, this study performed an additional 15 groups of calculations at $d=0.025 \mathrm{~m}, d=0.05 \mathrm{~m}, d=0.15 \mathrm{~m}, d=0.25 \mathrm{~m}$, and $d=0.40 \mathrm{~m}$, obtaining the following conclusions: $n$ gradually decreases with the increase of $d ; n$ takes the minimum value when $d=0.20 \mathrm{~m} ; n$ increases with $d$ when $d>0.20 \mathrm{~m}$ and when $d>0.30 \mathrm{~m}, n$ tends to be stable at about $0.03 \mathrm{~m}$, as shown in Figure 6c.

(4) Taking the height of the prism $H$ as the variable

Similarly, since the relationship between $H$ and $n$ is not obvious, this study performed an additional 15 groups of calculations at $H=0.10 \mathrm{~m}, H=0.20 \mathrm{~m}, H=0.40 \mathrm{~m}, H=0.50 \mathrm{~m}$, and $H=0.70 \mathrm{~m}$, obtaining the following conclusions: $n$ gradually decreases with the increase of $H ; n$ takes the minimum value when $H=0.50 \mathrm{~m}$; and $n$ increases with the increase of $H$ when $H>0.50 \mathrm{~m}$, as shown in Figure $6 \mathrm{~d}$.

To conclude, the optimal model parameters with the highest accuracy for slope deformation measurement are as follows: interval of scanning points $t=0.05 \mathrm{~m}$, radius of the sphere used for normal estimation $R=0.20 \mathrm{~m}$, side length of the prism $d=0.20 \mathrm{~m}$, and height of the prism $H=0.50 \mathrm{~m}$. 


\section{Case Study}

\subsection{Research Object}

The research object was the left side of the K146 + 150 point at S236 Boshan section, Shandong Province. The gradient was about $80^{\circ}$, the aspect was about $110^{\circ}$, the relative height difference was about $30 \mathrm{~m}$, and the width was about $94.5 \mathrm{~m}$. Cambrian limestone was exposed on the surface and there was almost no vegetation coverage. The location of the research object is shown in Figure 7.

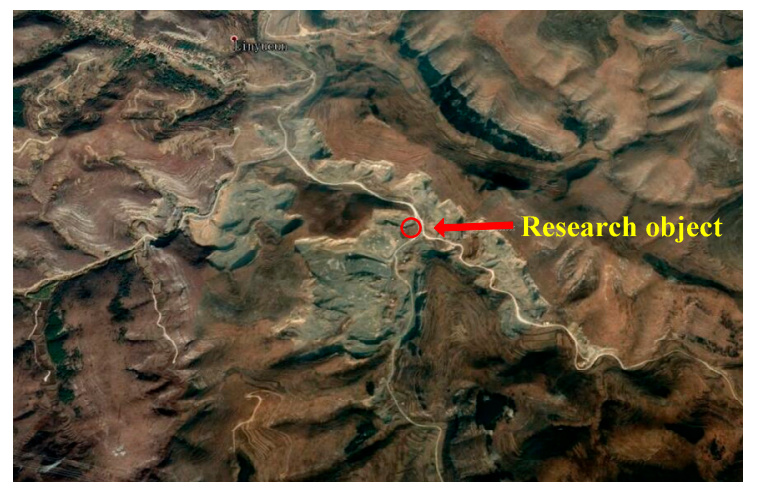

Figure 7. The location of the research object.

From 4-6 July 2018, Boshan district had been hit by severe rainstorms, rockfall disasters had occurred on the research object, and a number of tension cracks at the top of the slope appeared to penetrate. Once a large-scale instability occurs, it may block S236 and threaten the lives and property of nearby residents and pedestrians.

Two-phase scanning of the research object was performed with a Leica HDS-8800 3D laser scanner in 14 August 2018 and 9 November 2019, respectively. In order to reduce errors and improve accuracy, the pouring position of the concrete pile on the open ground opposite the research object was determined by using total station measurements. Data were collected once a week for a total of four times. The data analysis results verified that the pouring position was fixed. A screw was used to fix the $3 \mathrm{~d}$ laser scanner on the pile and the coordinate system used was the coordinate system of the $3 \mathrm{~d}$ laser scanner itself [37]. The scanning process included two steps: coarse scanning and fine scanning [53]. The coarse scanning and fine scanning both used two stations to obtain the whole slope information. Of which, the purpose of coarse scanning was to estimate the scanning time and determine whether the whole slope could be identified [54]. The interval of scanning point was set to $0.20 \mathrm{~m}$ for a total of 53,385 points. The purpose of fine scanning was to provide the point cloud data required for deformation measurement [55]. The interval of scanning points was $0.05 \mathrm{~m}$, as determined in Section 3 in this paper, and a total of 851,467 points were scanned.

\subsection{Calculation Results}

Point cloud data registration was carried out using the method proposed in Section 2 of this paper. The total time taken was $34.7 \mathrm{~s}$, and the registration results are shown in Figure 8.

Taking $R=0.20 \mathrm{~m}, d=0.20 \mathrm{~m}$, and $H=0.50 \mathrm{~m}$ as the model parameters, the slope deformation calculations were carried out using the method proposed in Section 3 of this paper, and the calculation results are shown in Figure 9, where positive values represent upward deformations in the normal direction, and negative values represent downward deformations in the normal direction $[45,47]$. 


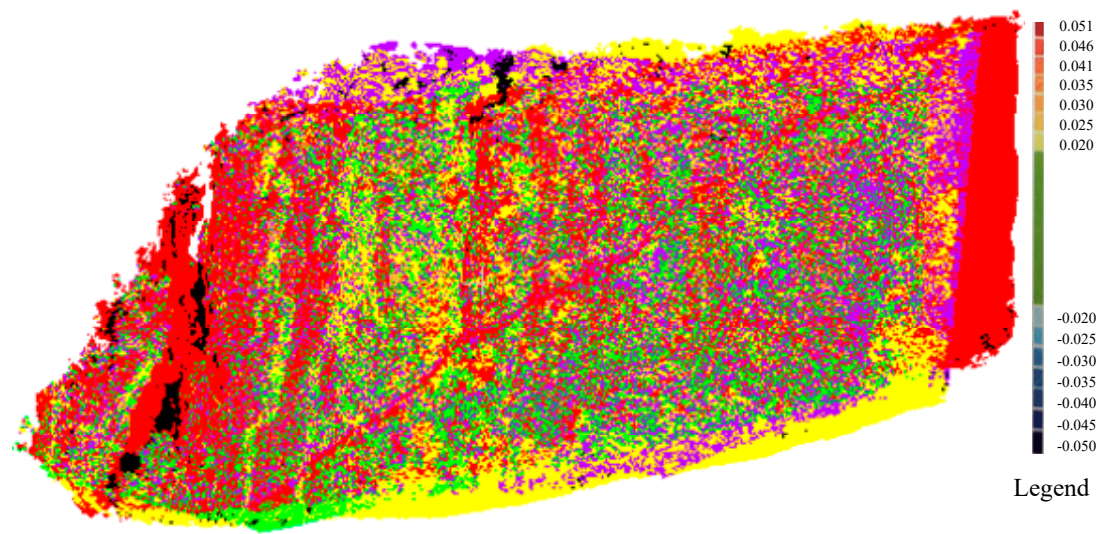

Figure 8. Registration results.

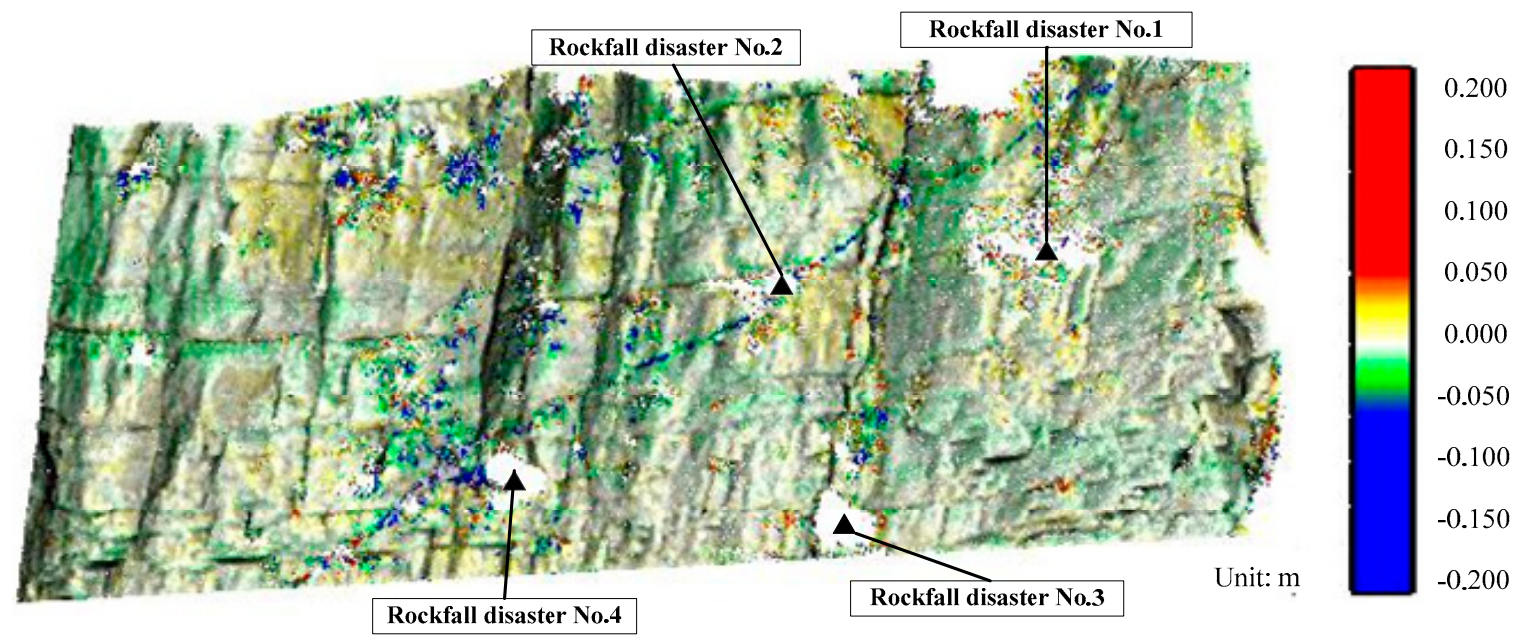

Figure 9. Slope deformation measurement results.

It can be seen from Figure 9 that from 14 August 2018 and 9 November 2019, a total of four rockfall disasters occurred at the wide cracks on the surface of the slope; the overall deformation of the slope was small with a maximum value of $0.183 \mathrm{~m}$ and occurred at the middle of the wide tension crack in the upper part of the slope. According to Li et al. [13] and Li et al. [56], the slope will continue to maintain a stable state without special inducing factors such as earthquake, heavy rainfall, and artificial excavation.

\section{Conclusions}

The coarse registration method of point cloud based on SIFT feature points and the fine registration method based on the K-D tree improved ICP algorithm were proposed. The results showed that they were superior to other algorithms (such as SURF feature points, Harris feature points, and LM improved ICP algorithm) when taking the Stanford Bunny as an example, and have high applicability in coarse and fine registration. In order to integrate the advantages of point measurement and surface measurement, an improved point cloud comparison method was proposed and the optimal model parameters were determined through model tests. A case study was conducted on the left side of the K146 + 150 point at S236 Boshan section, Shandong Province, where research results showed that from 14 August 2018 and 9 November 2019, the overall deformation of the slope was small with a maximum value of $0.183 \mathrm{~m}$, and the slope will continue to maintain a stable state without special inducing factors such as earthquake, heavy rainfall, and artificial excavation. 
Author Contributions: C.Y. and H.L. initiated and developed the concepts related to this research work; C.Y. and Y.L. proposed the point cloud registration method; C.Y. and Z.H. proposed the improved point cloud comparison method; C.Y. wrote the paper draft under Li's guidance. All authors discussed the results and commented on the manuscript. All authors have read and agreed to the published version of the manuscript.

Acknowledgments: This research was supported by the National Natural Science Foundation of China (Grant No. 51808327) and the Natural Science Foundation of Shandong Province (Grant No. ZR2019PEE016).

Conflicts of Interest: The authors declare no conflict of interest.

\section{References}

1. Liu, J.X. Simulating quasi-brittle failures including damage-induced softening based on the mechanism of stress redistribution. Appl. Math. Model. 2018, 55, 685-697. [CrossRef]

2. Codeglia, D.; Dixon, N.; Fowmes, G.J.; Marcato, G. Analysis of acoustic emission patterns for monitoring of rock slope deformation mechanisms. Eng. Geol. 2017, 219, 21-31. [CrossRef]

3. Kasprzak, M.; Jancewicz, K.; Różycka, M.; Kotwicka, W. Geomorphology- and geophysics-based recognition of stages of deep-seated slope deformation (Sudetes, SW Poland). Eng. Geol. 2019, 260, 105230. [CrossRef]

4. Yin, C. Hazard assessment and regionalization of highway flood disasters in China. Nat. Hazards 2020, 100, 535-550. [CrossRef]

5. Nie, Z.B.; Zhang, Z.H.; Zheng, H. Slope stability analysis using convergent strength reduction method. Eng. Anal. Bound. Elem. 2019, 108, 402-410. [CrossRef]

6. Jiang, Q.; Yan, F.; Wu, J.; Fan, Q.; Li, S.; Xu, D. Grading opening and shearing deformation of deep outward-dip shear belts inside high slope: A case study. Eng. Geol. 2019, 250, 113-129. [CrossRef]

7. Yin, C.; Zhang, J.L. Hazard regionalization of debris-flow disasters along highways in China. Nat. Hazards 2018, 91, 129-147. [CrossRef]

8. Riccardi, U.; Arnoso, J.; Benavent, M.; Vélez, E.; Tammaro, U.; Montesinos, F.G. Exploring deformation scenarios in Timanfaya volcanic area (Lanzarote, Canary Islands) from GNSS and ground based geodetic observations. J. Volcanol. Geotherm. Res. 2018, 357, 14-24. [CrossRef]

9. Yang, Y.; Zheng, Y.; Yu, W.K.; Chen, W.; Weng, D.J. Deformation monitoring using GNSS-R technology. Adv. Space Res. 2019, 63, 3303-3314. [CrossRef]

10. Beregovoi, D.V.; Younes, J.A.; Mustafin, M.G. Monitoring of quarry slope deformations with the use of satellite positioning technology and unmanned aerial vehicles. Procedia Eng. 2017, 189, 737-743. [CrossRef]

11. Zhang, S.; Wang, H.; Gao, J.G.; Xing, C.Q. Frequency domain point cloud registration based on the Fourier transform. J. Vis. Commun. Image Represent. 2019, 61, 170-177. [CrossRef]

12. Xu, X.Y.; Bureick, J.; Yang, H.; Neumann, I. TLS-based composite structure deformation analysis validated with laser tracker. Compos. Struct. 2018, 202, 60-65. [CrossRef]

13. Li, C.D.; Fu, Z.Y.; Wang, Y.; Tang, H.M.; Yan, J.F.; Gong, W.P.; Yao, W.M.; Robert, E.C. Susceptibility of reservoir-induced landslides and strategies for increasing the slope stability in the Three Gorges Reservoir Area: Zigui Basin as an example. Eng. Geol. 2019, 261, 105279. [CrossRef]

14. Xu, X.Y.; Kargoll, B.; Bureick, J.; Yang, H.; Alkhatib, H.; Neumann, I. TLS-based profile model analysis of major composite structures with robust B-spline method. Compos. Struct. 2018, 184, 814-820. [CrossRef]

15. Quan, S.; Ma, J.; Hu, F.; Fang, B.; Ma, T. Local voxelized structure for 3D binary feature representation and robust registration of point clouds from low-cost sensors. Inf. Sci. 2018, 444, 153-171. [CrossRef]

16. Song, Z.P.; Cheng, Y.; Tian, X.X.; Wang, J.B.; Yang, T.T. Mechanical properties of limestone from Maixi tunnel under hydro-mechanical coupling. Arab. J. Geosci. 2020, in press.

17. Chang, S.; Ahn, C.; Lee, M.; Oh, S. Graph-matching-based correspondence search for nonrigid point cloud registration. Comput. Vis. Image Underst. 2020, 192, 102899. [CrossRef]

18. Zhu, Y.X.; Cheng, S.; Stanković, V.; Stanković, L. Image registration using BP-SIFT. J. Vis. Commun. Image Represent. 2013, 24, 448-457. [CrossRef]

19. Chen, Y.; Shang, L. Improved SIFT image registration algorithm on characteristic statistical distributions and consistency constraint. Optik 2016, 127, 900-911. [CrossRef]

20. Wang, G.; Shi, Z.C.; Shang, Y.; Yu, Q.F. Automatic extraction of diagonal markers based on template matching and peaks of gradient histogram. ACTA Opt. Sin. 2018, 38, 0815014. (In Chinese) [CrossRef] 
21. Lu, T.D.; Yuan, Z.C.; Zheng, K. Super 4PCS Point Cloud Registration Algorithm Combining Scale Invariant Features. Remote Sens. Inf. 2019, 34, 15-20. (In Chinese)

22. Ran, Y.Y.; Xu, X.B. Point cloud registration method based on SIFT and geometry feature. Optik 2020, 203, 163902. [CrossRef]

23. Besl, P.J.; McKay, H.D. A method for registration of 3-D shapes. IEEE Trans. Pattern Anal. Mach. Intell. 1992, 14, 239-256. [CrossRef]

24. Shi, X.Y.; Peng, J.J.; Li, J.P.; Yan, P.T.; Gong, H.Y. The Iterative Closest Point Registration Algorithm Based on the Normal Distribution Transformation. Procedia Comput. Sci. 2019, 147, 181-190. [CrossRef]

25. Hermans, J.; Smeets, D.; Vandermeulen, D.; Suetens, P. Robust point set registration using EM-ICP with information-theoretically optimal outlier handling. In Proceedings of the IEEE Conference Computer Vision and Pattern Recognition, Providence, RI, USA, 20-25 June 2011; pp. 2465-2472.

26. Zeng, F.X.; Li, L.; Diao, X.P. Iterative closest point algorithm registration based on curvature features. Laser Optoelectron. Prog. 2017, 54, 011003. (In Chinese) [CrossRef]

27. Wei, S.B.; Wang, S.Q.; Zhou, C.H.; Liu, K.; Fan, X. An Iterative Closest Point Algorithm Based on Biunique Correspondence of Point Clouds for 3D Reconstruction. ACTA Opt. Sin. 2015, 35, 0515003. (In Chinese)

28. Li, W.; Song, P. A modified ICP algorithm based on dynamic adjustment factor for registration of point cloud and CAD model. Pattern Recognit. Lett. 2015, 65, 88-94. [CrossRef]

29. Cheng, S.; Marras, I.; Zafeiriou, S.; Pantic, M. Statistical non-rigid ICP algorithm and its application to 3D face alignment. Image Vis. Comput. 2017, 58, 3-12. [CrossRef]

30. Wu, Z.Z.; Chen, H.C.; Du, S.Y.; Fu, M.Y.; Zhou, N.; Zheng, N.N. Correntropy based scale ICP algorithm for robust point set registration. Pattern Recognit. 2019, 93, 14-24. [CrossRef]

31. Combès, B.; Prima, S. An efficient EM-ICP algorithm for non-linear registration of large 3D point sets. Comput. Vis. Image Underst. 2020, 191, 102854. [CrossRef]

32. Assefa, S.; Graziani, A.; Lembo-Fazio, A. A slope movement in a complex rock formation: Deformation measurements and DEM modeling. Eng. Geol. 2016, 219, 74-91. [CrossRef]

33. Jo, H.C.; Kim, J.; Lee, K.; Sohn, H.G.; Lim, Y.M. Non-contact strain measurement for laterally loaded steel plate using LiDAR point cloud displacement data. Sens. Actuators A Phys. 2018, 283, 362-374. [CrossRef]

34. Cho, Y.C.; Song, Y.S. Deformation measurements and a stability analysis of the slope at a coal mine waste dump. Ecol. Eng. 2014, 68, 189-199. [CrossRef]

35. Schürch, P.; Densmore, A.L.; Rosser, N.J.; Lim, M. Detection of surface change in complex topography using terrestrial laser scanning: Application to the Illgraben debris-flow channel. Earth Surf. Process. Landf. 2011, 36, 1847-1859. [CrossRef]

36. Zhu, H.X.; Sui, L.C.; Lu, K.X. Application of terrestrial 3D laser scanning technology in deformation monitoring of dangerous rock mass. Bull. Surv. Mapp. 2017, 11, 68-71. (In Chinese)

37. Hu, M.; Xie, M.W.; Xu, B. A method of fixed-point monitoring of landslide deformation based on 3D laser scanning. Journal of Southwest University. Nat. Sci. Ed. 2017, 39, 155-164. (In Chinese)

38. Dong, Z.; Yang, B.S.; Liang, F.X.; Huang, R.G. Hierarchical registration of unordered TLS point clouds based on binary shape context descriptor. ISPRS J. Photogramm. Remote Sens. 2018, 61, 61-79. [CrossRef]

39. Zhang, Y.W.; Song, Z.P.; Weng, X.L.; Xie, Y.L. A new soil-water characteristic curve model for unsaturated loess based on wetting-induced pore deformation. Geofluids 2019, 2019, 1672418. [CrossRef]

40. Zhang, J.; Xiu, X.J. K-d tree based approach for point location problem in explicit model predictive control. J. Frankl. Inst. 2018, 355, 5431-5451.

41. Chen, Y.W.; Zhou, L.D.; Tang, Y.; Singh, J.P.; Bouguila, N.; Wang, C.; Wang, H.Z.; Du, J.X. Fast neighbor search by using revised k-d tree. Inf. Sci. 2019, 472, 145-162. [CrossRef]

42. Song, Z.P.; Mao, J.C.; Tian, X.X.; Zhang, Y.W.; Wang, J.B. Optimization analysis of controlled blasting for passing through houses at close range in super-large section tunnels. Shock Vib. 2019, 2019, 1941436. [CrossRef]

43. Zhou, S.T.; Liu, X.L.; Wang, C.Y.; Yang, B. Non-iterative denoising algorithm based on a dual threshold for a 3D point cloud. Opt. Lasers Eng. 2020, 126, 105921. [CrossRef]

44. Wu, Y.F.; Wang, W.; Lu, K.Q.; Wei, Y.D.; Chen, Z.C. A New Method for Registration of 3D Point Sets with Low Overlapping Ratios. Procedia CIRP 2015, 27, 202-206. [CrossRef]

45. Acikgoz, S.; Soga, K.; Woodhams, J. Evaluation of the response of a vaulted masonry structure to differential settlements using point cloud data and limit analyses. Constr. Build. Mater. 2017, 150, 916-931. [CrossRef] 
46. Gao, Y.; Wu, P.; Li, W.; Xuan, Y.L.; Hou, X.D. Simultaneous and selective preconcentration of trace Cu and Ag by one-step displacement cloud point extraction for FAAS determination. Talanta 2010, 81, 586-590. [CrossRef]

47. Kamnik, R.; Perc, M.N.; Topolšek, D. Using the scanners and drone for comparison of point cloud accuracy at traffic accident analysis. Accid. Anal. Prev. 2020, 135, 105391. [CrossRef] [PubMed]

48. Li, Y.; Yong, B.; Oosterom, P.V.; Lemmens, M.; Wu, H.Y.; Ren, L.L.; Zheng, M.X.; Zhou, J.J. Airborne LiDAR Data Filtering Based on Geodesic Transformations of Mathematical Morphology. Remote Sens. 2017, 9, 1104. [CrossRef]

49. Lim, T.W.; Charles, E.O. Model-free pose estimation using point cloud data. Acta Astronaut. 2019, 165, $298-311$. [CrossRef]

50. Dai, W.X.; Yang, B.S.; Liang, X.L.; Dong, Z.; Huang, R.G.; Wang, Y.S.; Li, W.Y. Automated fusion of forest airborne and terrestrial point clouds through canopy density analysis. ISPRS J. Photogramm. Remote Sens. 2019, 156, 94-107. [CrossRef]

51. Zhen, Z.; Xuan, Z.M.; Wang, F.; Sun, R.F.; Duić, N.; Jin, T. Image phase shift invariance based multi-transform-fusion method for cloud motion displacement calculation using sky images. Energy Convers. Manag. 2019, 197, 111853. [CrossRef]

52. Ma, J.W.; Czerniawski, T.; Leite, F. Semantic segmentation of point clouds of building interiors with deep learning: Augmenting training datasets with synthetic BIM-based point clouds. Autom. Constr. 2020, 113, 103144. [CrossRef]

53. Zhang, W.M.; Chen, Y.M.; Wang, H.T.; Chen, M.; Wang, X.Y.; Yan, G.J. Efficient registration of terrestrial LiDAR scans using a coarse-to-fine strategy for forestry applications. Agric. For. Meteorol. 2016, 225, 8-23. [CrossRef]

54. Olmos, P. Coarse approximation to the multiresolution response of a fiber optic intensity-based scanning 3D-microscope. Opt. Fiber Technol. 2017, 37, 43-52. [CrossRef]

55. Chang, J.W.; Lin, S.F.; Hsu, C.Y. Accurate and rapid alignment of laser scanned 3D surface using TSK-type neural-fuzzy network-based coarse-to-fine strategy. Opt. Lasers Eng. 2012, 50, 1450-1458. [CrossRef]

56. Li, X.W.; Zhu, J.Q.; Li, Z.W.; Yang, X.L. 3D stability assessment of stepped slopes in inhomogeneous soils. J. Cent. South Univ. 2020, 27, 221-230. [CrossRef] 